\title{
A Deconvolution Approach for the Mapping of Acoustic Sources (DAMAS) Determined from Phased Microphone Arrays
}

\author{
Thomas F. Brooks* \\ William M. Humphreys, Jr. ${ }^{\dagger}$ \\ NASA Langley Research Center \\ Hampton, Virginia 23681-0001
}

\begin{abstract}
Current processing of acoustic array data is burdened with considerable uncertainty. This study reports an original methodology that serves to demystify array results, reduce misinterpretation, and accurately quantify position and strength of acoustic sources. Traditional array results represent noise sources that are convolved with array beamform response functions, which depend on array geometry, size (with respect to source position and distributions), and frequency. The Deconvolution Approach for the Mapping of Acoustic Sources (DAMAS) method removes beamforming characteristics from output presentations. A unique linear system of equations accounts for reciprocal influence at different locations over the array survey region. It makes no assumption beyond the traditional processing assumption of statistically independent noise sources. The full rank equations are solved with a new robust iterative method. DAMAS is quantitatively validated using archival data from a variety of prior high-lift airframe component noise studies, including flap edge/cove, trailing edge, leading edge, slat, and calibration sources. Presentations are explicit and straightforward, as the noise radiated from a region of interest is determined by simply summing the mean-squared values over that region. DAMAS can fully replace existing array processing and presentations methodology in most applications. It appears to dramatically increase the value of arrays to the field of experimental acoustics.
\end{abstract}

\footnotetext{
*Senior Research Scientist, Aeroacoustics Branch, Associate Fellow AIAA.

${ }^{\dagger}$ Senior Research Scientist, Aeroacoustics Branch, Senior Member AIAA.

Copyright (C) 2004 by the American Institute of Aeronautics and Astronautics, Inc. No copyright is asserted in the United States under Title 17, U.S. Code. The U.S. Government has a royalty-free license to exercise all rights under the copyright claimed herein for government purposes. All other rights are reserved by the copyright owner.
}

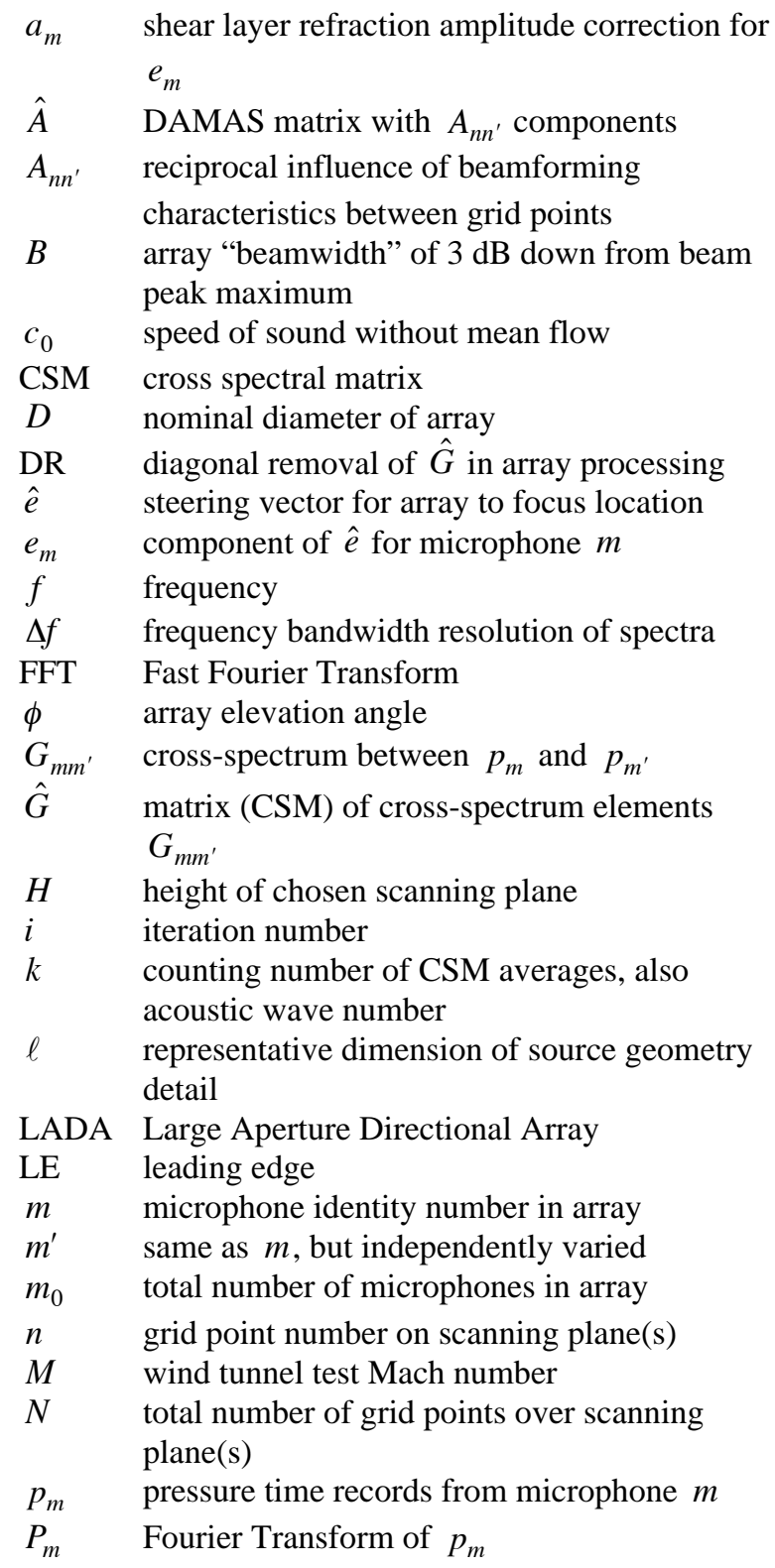




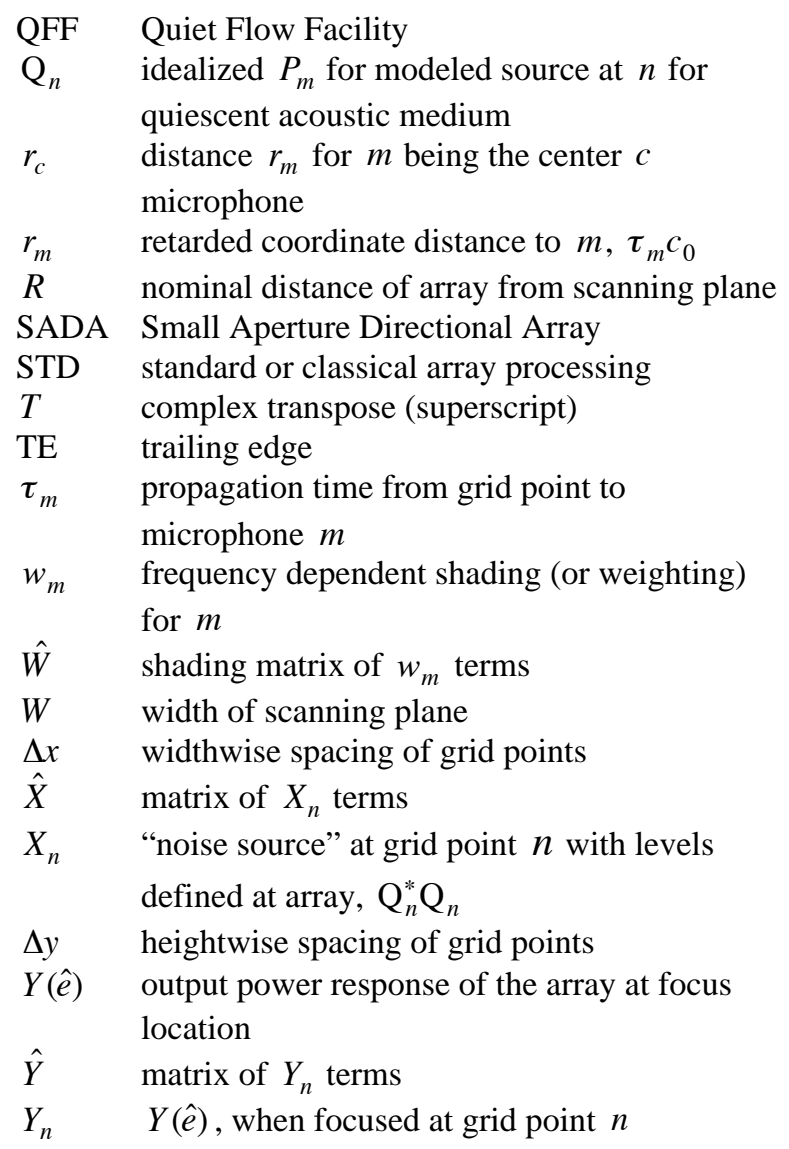

Subscripts

$\begin{array}{ll}b k g & \text { background } \\ \text { diag } & \text { diagonal } \\ m: n & \text { term associated with } m, \text { as it relates to grid } \\ & \text { position } n \\ \text { mod } & \text { modeled }\end{array}$

\section{INTRODUCTION}

Over recent years, particularly since the mid 1990 's, the use of phased arrays of microphones in the study of aeroacoustic sources has greatly increased. Its popularity is due in large part to the seemingly magical presentations of array-processed results, which pull out features (real or just perceived) of noise source distributions on both wind tunnel models and full-scale aircraft. Properly utilized, arrays have been powerful tools that can often be used to extract noise source radiation information in circumstances where other measurement techniques may fail. However, presentations of array measurements of aeroacoustic noise sources can lend themselves to great uncertainty in interpretation. Proper interpretation requires knowledge of the principles of phased arrays and processing methodology. Even then, because of the complexity and differing processing philosophy, there can be misinterpretations of actual source distributions (and subsequent misdirection of engineering efforts).

Before the mid 1980's for aeroacoustic studies, processing of array microphone signals involved time delay shifting of signals and summing in order to strengthen contributions from, and thus "focus" on, chosen locations over surfaces or positions in the flow field. Over the years, with great advances in computers, this basic "delay and sum" processing approach has been replaced by "classical beamforming" approaches involving spectral processing to form cross spectral matrices (CSM) and phase shifting using increasingly large array element numbers. Such advances have greatly increased productivity and processing flexibility, but have not changed at all the interpretation complexity of the processed array results. Brooks and Humphreys ${ }^{1}$ examined current capabilities and determined the role of array size on the quantitative measurement of airframe noise sources.

At Langley Research Center, for testing in the Quiet Flow Facility (QFF), quantitative definition of different airframe noise sources spectra and directivity has been a primary goal. This goal has been achieved with arrays in a rather straight forward manner for the localized intense source of flap edge noise ${ }^{2}$; although for precise source localization, Coherent Output Power (COP) methods were used by incorporating unsteady surface pressure measurements along with the array. Quantitative measurements for distributed sources of slat noise ${ }^{3}$ were achieved using an array and specially tailored weighting functions that matched array beampatterns with knowledge of the line source type distribution for slat noise. Similar measurements for distributed trailing edge noise $e^{4,5}$ and leading edge noise (due in this case to grit boundary layer tripping) were made along with special COP methodologies involving microphone groups.

Outside of Langley, there have been a number of efforts to analyze and develop more effective array processing methodologies in order to more readily extract source information. Several efforts include those to better account for array resolution, ray path coherence loss, and source distribution coherence ${ }^{6}$ and for test rig reflections ${ }^{7}$. In a simulation study ${ }^{8}$ of methods for improving array output, particularly for suppressing sidelobe contamination, three beamforming techniques were examined. These were a cross spectral matrix (CSM) element weighting approach, a robust adaptive beamforming, and the CLEAN algorithm. The CSM weighting approach 
reduced sidelobes compared to classical beamforming with some overall improvement in main resolution. The results for the adaptive beamformer, used with a specific constant added to the CSM matrix diagonal to avoid instability problems, were encouraging. The CLEAN algorithm was found to have the best overall performance for the simulated beamforming exercise. CLEAN is a deconvolution technique first used in radio astronomy ${ }^{9}$. It was also examined in another study $^{10}$, along with a related algorithm called RELAX $^{11}$, using Langley QFF experimental array calibration data for a no-flow condition. It found mixed success in separating out sources. In a different study ${ }^{12}$, using the same data, two robust adaptive beamforming methods were examined and found to be capable of giving sharp beamwidths and low sidelobes. It should be mentioned that the above methods, although perhaps offering promise, have not been demonstrated to produce quantitatively accurate source amplitudes and distributions for real test cases. In the particular method of CLEAN, questions have been raised ${ }^{13}$ with regard to practicality of the algorithm for arrays in reflective wind tunnel environments. A method that did show promise with wind tunnel aeroacoustic data is the Spectral Estimation Method ${ }^{14}$ (SEM). The method requires that the measured CSM of the array be compared to a simulated CSM constructed by defining distributions of compact patches of sources (or source areas) over a chosen aeroacoustic region of interest. The difference between the two CSMs is minimized using a Conjugate Gradient Method. The application of positivity constraints on the source solutions was found to be difficult. The resultant source distributions for the airframe noise cases examined were regarded as being feasible and realistic, although not unique.

The goal of the present effort was to develop a complete deconvolution approach for the mapping of acoustic sources (DAMAS ${ }^{15}$ ) to demystify 2D and 3D array results, to reduce misinterpretation, and to more accurately quantify position and strength of aeroacoustic sources. Traditional presentations of array results involve mapping (contour plotting) of array output over spatial regions. These maps do not truly represent noise source distributions, but ones that are convolved with the array response functions, which depend on array geometry, size (with respect to source position and distributions), and frequency. The deconvolution methodology presented in this paper employs these processed results (array output at grid points) over the survey regions and the associated array beamforming characteristics (relating the reciprocal influence of the different grid point locations) over the same regions where the array's outputs are measured. A linear system of " $N$ " (number of grid points in region) equations and " $N$ " unknowns is created. These equations are solved in a straight-forward iteration approach. The end result of this effort is a unique robust deconvolution algorithm designed to determine the "true" noise source distribution over an aeroacoustic source region to replace the classical beamformed distributions. Example applications include ideal point and line noise source cases, well as conformation with well documented experimental airframe noise studies of wing trailing and leading edge noise, slat noise, and flap edge / flap cove noise.

\section{DAMAS ANALYSIS}

Beamforming. The first step in the analysis is to beamform over the source region, using what have become traditional methods. Post processing of simultaneously acquired data from the microphones of an array begins with computation of the cross-spectral matrix for each test case data set. The computation of each element of the matrix is performed using Fast Fourier Transforms (FFT) of the original data ensemble. The transform pairs $P_{m}(f, \mathrm{~T})$ and $P_{m^{\prime}}(f, \mathrm{~T})$ are formed from pressure time records $p_{m}(\mathrm{t})$ and $p_{m^{\prime}}(\mathrm{t})$, defined at discrete sampling times that are $\Delta \mathrm{t}$ apart, of data block lengths $\mathrm{T}$ from microphones $m$ and $m^{\prime}$, respectively. The cross-spectrum matrix element is ${ }^{1}$

$$
G_{m m^{\prime}}(f)=\frac{2}{K \mathrm{w}_{\mathrm{s}} \mathrm{T}} \sum_{k=1}^{K}\left[P_{m k}^{*}(f, \mathrm{~T}) P_{m^{\prime} k}(f, \mathrm{~T})\right]
$$

This one-sided cross-spectrum is averaged over $K$ block averages. The total record length is $\mathrm{T}_{t o t}=K \mathrm{~T}$. The term $\mathrm{w}_{\mathrm{s}}$ is a data-window (such as Hamming) weighting constant. $G_{m m^{\prime}}(f)$ is seen to be a complex spectrum with values at discrete frequencies $f$, which are $\Delta f$ apart. The bandwidth is $\Delta f=1 / \mathrm{T}(\mathrm{Hz})$. The full matrix is, with $m_{0}$ being the total number of microphones in the array,

$$
\hat{G}=\left[\begin{array}{cccc}
G_{11} & G_{12} & \cdots & G_{1 m_{0}} \\
\vdots & G_{22} & & \vdots \\
\vdots & & \ddots & \vdots \\
G_{m_{0} 1} & & & G_{m_{0} m_{0}}
\end{array}\right]
$$

Note that the lower triangular elements are complex conjugates of the upper triangular elements.

The cross-spectral matrix is employed in conventional beamforming approaches to electronically 


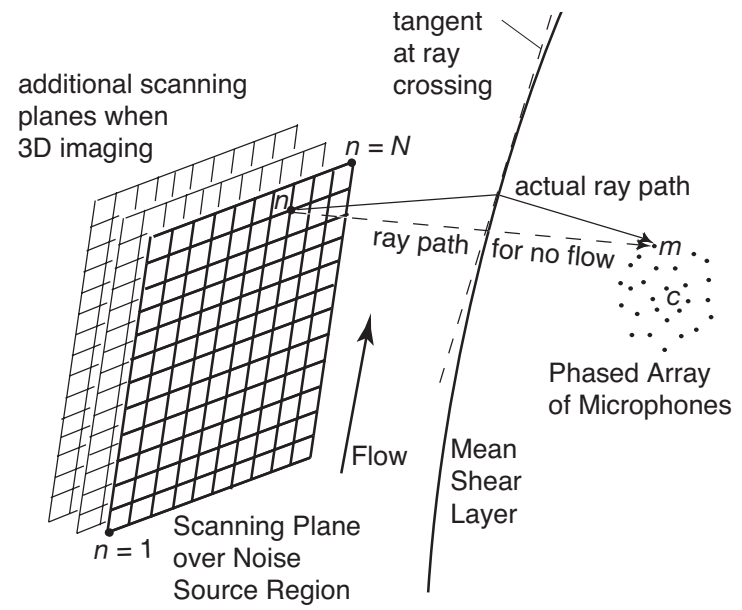

FIGURE 1 Illustration of open jet test configuration where the array is out of flow and the scanning plane positioned over aeroacoustic source region.

"steer" to chosen noise source locations about an aeroacoustic test model. Figure 1 illustrates a particular test setup of a distribution of microphones of a phased array located outside the flow field containing an aeroacoustic model. A scanning plane of grid points is defined over the noise source region. (A scanning plane may, for example, be placed through the chordline of an airfoil section when studying trailing edge and/or leading edge noise.) The beamforming approach involves steering vectors associated with each microphone with respect to the chosen steering location. In Fig. 1, the steering location is designated as grid point $n$. The steering vector is

$$
\hat{e}=\operatorname{col}\left[\begin{array}{llll}
e_{1} & e_{2} & \cdots & e_{m_{0}}
\end{array}\right]
$$

where the component for each microphone $m$ is

$$
e_{m}=a_{m} \frac{r_{m}}{r_{c}} \exp \left\{j 2 \pi f \tau_{m}\right\}
$$

The vector components serve to phase shift each microphone signal to allow constructive summing of contributions from the chosen locations. $\tau_{m}$ is the time required to propagate from grid point $n$ to microphone $m$. In Ref. 1, the phase is designated as

$$
2 \pi f \tau_{m}=\left(\vec{k} \cdot \vec{x}_{m}\right)+2 \pi f \Delta \mathrm{t}_{m, \text { shear }}
$$

The term $\vec{k}$ is the acoustic wave vector, $\vec{x}_{m}$ is the distance vector from the steering location to the microphone $m$. The steering vector components contain terms that account for the mean amplitude and phase changes due to convected and refracted sound transmission through the shear layer to each microphone. The corrections are calculated ${ }^{16}$ by the use of Snell's law in Amiet's method ${ }^{17}$, adapted to a curved three-dimensional mean shear layer surface defined in the shear layer. $a_{m}$ is the refraction amplitude correction. $\Delta \mathrm{t}_{m \text {,shear }}$ is the additional time (compared to a direct ray path with no flow) it takes an acoustic ray to travel to microphone $m$ from the steering location $n$, due to the convection by the open jet flow and refraction by the shear layer. In Eq. (4), the ratio $\left(r_{m} / r_{C}\right)$ is included to normalize the distance related amplitude to that of the distance $r_{C}$ from the source location to the array center microphone at $c$. Both $r_{m}$ and $r_{C}$ are in terms of "retarded" coordinates ${ }^{5,16}$. With this, $r_{m}=\tau_{m} c_{0}$, where $c_{0}$ equals the speed of sound without mean flow.

For classical or standard (STD) array beamforming, the output power spectrum (or response) of the array is obtained from

$$
Y(\hat{e})=\frac{\hat{e}^{T} \hat{G} \hat{e}}{m_{0}^{2}}
$$

where the superscript $T$ denotes a complex transpose of the steering vector. Here $Y(\hat{e})$ is a mean-pressuresquared per frequency bandwidth quantity. The division by the number of array microphones squared serves to reference levels to that of an equivalent single microphone measurement. Note that the cross-spectral matrix (CSM) $\hat{G}$ often has a corresponding background cross-spectral matrix $\hat{G}_{b k g}$ (obtained for a similar test condition except that the model is removed) subtracted from it to improve fidelity ${ }^{1}$.

Shading algorithms can be used over distributions of array microphones to modify the output beampattern. The shaded steered response is

$$
Y(\hat{e})=\frac{\hat{e}^{T} \hat{W} \hat{G} \hat{W}^{T} \hat{e}}{\left(\sum_{m=1}^{m_{0}} w_{m}\right)^{2}}
$$

where $w_{m}$ is the frequency dependent shading (or weighting) for each microphone $m . \hat{W}$ is a row matrix containing the $w_{m}$ terms. When all $w_{m}$ terms are set to one and $W$ becomes an identity matrix, all microphones are fully active in the beamforming to render Eq. (6). 
(In Refs. 2-5 and 18,19, a special shading is used to maintain constant beamwidth over a range of frequencies by shading out $\left(w_{m}=0\right)$ inner microphone groups at low frequencies and by shading out outer groups at high frequencies.)

A modified form of Eq. (6) is often used to improve dynamic range of the array results in poor signal-to-noise test applications. The primary intent is to remove the microphone self noise contamination (particularly caused by turbulence interacting with the microphones). This is done by removing (zeroing out) the diagonal terms of $\hat{G}$ and accounting for this change in the number of terms of $\hat{G}$ in the denominator. The output of Diagonal Removal (DR) processing is

$$
Y(\hat{e})=\frac{\hat{e}^{T} \hat{G}_{\text {diag }=0} \hat{e}}{m_{0}^{2}-m_{0}}
$$

This modifies the beamform patterns compared to Eq. (6). The diagonal can be viewed as expendable ${ }^{13}$ in the sense that it duplicates information contained in the cross terms of $\hat{G}$. However, great care must be taken in physical interpretation of resulting array response maps - for example, negative "pressure-squared" values are to be expected over low-level noise source regions. The corresponding shaded version of Eq. (8) is

$$
Y(\hat{e})=\frac{\hat{e}^{T} \hat{W} \hat{G}_{\text {diag }=0} \hat{W}^{T} \hat{e}}{\left(\sum_{m=1}^{m_{0}} w_{m}\right)^{2}-\left(\sum_{m=1}^{m_{0}} w_{m}\right)}
$$

The common practice for studying aeroacoustic source of noise with arrays are to determine the array response, using either Eq. (6), (7), (8), or (9), over a range (grid) of steering locations about the source region. For particular frequencies, contours of the response levels are plotted over planes where sources are know to lie, or over volume regions in some cases. To extract quantitative contributions to the noise field from particular source locations, a number of methods are used. Integration methods, such as that of Ref. 1, can be used as well as special methods tailored to fit particular noise distributions, such as in Refs. 3 - 5 . Still the methods can be difficult to apply and care must be taken in interpretation. This is because the processing of Eqs. (6)-(9) produces "source" maps which are as much a reflection of the array beamforming pattern characteristics as is the source distribution being measured.
DAMAS Inverse Problem Definition. The purpose here is to pose the array problem such that the desired quantities, the source strength distributions, are extracted cleanly from the beamforming array characteristics. First, the pressure transform $P_{m}$ of microphone $m$ of Eq. (1) is related to a modeled source located at position $n$ in the source field.

$$
P_{m: n}=\mathrm{Q}_{n} e_{m: n}^{-1}
$$

Here $\mathrm{Q}_{n}$ represents the pressure transform that $P_{m: n}$ (or $P_{m}$ ) would be if flow convection and shear layer refraction did not affect transmission of the noise to microphone $m$, and if $m$ were at a distance of $r_{c}$ from $n$ rather than $r_{m}$. The $e_{m: n}^{-1}$ term is simply those things that are postulated in Eq. (4) to affect the signal in the actual transmission to render $P_{m}$. The product of pressure-transform terms of Eq. (1) becomes

$$
\begin{aligned}
P_{m: n}{ }^{*} P_{m^{\prime}: n} & =\left(\mathrm{Q}_{n} e_{m: n}^{-1}\right)^{*}\left(\mathrm{Q}_{n} e_{m^{\prime}: n}^{-1}\right) \\
& =\mathrm{Q}_{n}{ }^{*} \mathrm{Q}_{n}\left(e_{m: n}^{-1}\right)^{*} e_{m^{\prime}: n}^{-1}
\end{aligned}
$$

When this is substituted into Eq. (1), one obtains the modeled microphone array cross-spectral matrix for a single source located at $n$

$$
\hat{G}_{n_{\text {mod }}}=X_{n}\left[\begin{array}{cccc}
\left(e_{1}^{-1}\right)^{*} e_{1}^{-1} & \left(e_{1}^{-1}\right)^{*} e_{2}^{-1} & \cdots & \left(e_{1}^{-1}\right)^{*} e_{m_{0}}^{-1} \\
\left(e_{2}^{-1}\right)^{*} e_{1}^{-1} & \left(e_{2}^{-1}\right)^{*} e_{2}^{-1} & & \vdots \\
& & \ddots & \vdots \\
& & & \left(e_{m_{0}}^{-1}\right)^{*} e_{m_{0}}^{-1}
\end{array}\right]_{n}
$$

where $X_{n}$ is the mean square pressure per bandwidth at each microphone $m$ normalized in level for a microphone at $r_{m}=r_{c}$. (A similar expression is used within the integration technique of Ref. 1.) It is now assumed that there are a number $N$ of statistically independent sources, each at different $n$ positions. One obtains for the total modeled cross-spectral matrix

$$
\hat{G}_{\text {mod }}=\sum_{n} \hat{G}_{n_{\text {mod }}}
$$

Employing this in Eq. (6),

$$
Y_{n_{\mathrm{mod}}}(\hat{e})=\left[\frac{\hat{e}^{T} \hat{G}_{\mathrm{mod}} \hat{e}}{m_{0}^{2}}\right]_{n}
$$




$$
Y_{n_{\text {mod }}}(\hat{e})=\frac{\hat{e}_{n}^{T} \sum_{n^{\prime}} X_{n^{\prime}}[]_{n^{\prime}} \hat{e}_{n}}{m_{0}^{2}}=\sum_{n^{\prime}} \frac{\hat{e}_{n}^{T}[]_{n^{\prime}} \hat{e}_{n}}{m_{0}^{2}} X_{n^{\prime}}
$$

where the bracketed term is that of Eq. (12). This can be shown to equal

$$
Y_{n_{\text {mod }}}(\hat{e})=\hat{A} X_{n}
$$

where the components of matrix $\hat{A}$ are

$$
A_{n n^{\prime}}=\frac{\hat{e}_{n}^{T}[]_{n^{\prime}} \hat{e}_{n}}{m_{0}^{2}}
$$

By equating $Y_{n_{\bmod }}(\hat{e})$ with processed $Y(\hat{e})$ from measured data, we have

$$
\hat{A} \hat{X}=\hat{Y}
$$

Equation (18), for $\hat{X}$, also applies for the cases of shaded standard, DR, and shaded DR beamforming, with components $A_{n n^{\prime}}$ of $\hat{A}$ becoming

$$
\begin{gathered}
A_{n n^{\prime}}=\frac{\hat{e}^{T} \hat{W}[]_{n^{\prime}} \hat{W}^{T} \hat{e}}{\left(\sum_{m=1}^{m_{0}} w_{m}\right)^{2}}, \\
A_{n n^{\prime}}=\frac{\hat{e}_{n}^{T}\left([]_{n^{\prime}}\right)_{\text {diag }=0} \hat{e}_{n}}{m_{0}^{2}-m_{0}},
\end{gathered}
$$

and

$$
A_{n n^{\prime}}=\frac{\hat{e}_{n}^{T} \hat{W}\left([]_{n^{\prime}}\right)_{\operatorname{diag}=0} \hat{W}^{T} \hat{e}_{n}}{\left(\sum_{m=1}^{m_{0}} w_{m}\right)^{2}-\left(\sum_{m=1}^{m_{0}} w_{m}\right)}
$$

respectively. For standard beamforming (shaded or not) the diagonal terms for $\hat{A}$ are equal to one. For Diagonal Removal beamforming (shaded or not), the diagonal terms for $\hat{A}$ are also equal to one, but the offdiagonal components differ and attain negative values when $n$ and $n^{\prime}$ represent sufficiently distant points from one another, depending on frequency.

Equation (18) represents a system of linear equations relating a spatial field of point locations, with beamformed array-output responses $Y_{n}$, to equivalent source distributions $X_{n}$ at the same point locations. The same is true of Eq. (18) when $Y_{n}$ is the result of shaded and/or DR processing of the same acoustic field. $X_{n}$ is the same in both cases. (One is not restricted to these particular beamforming processing as long as $\hat{A}$ is appropriately defined.) Equation (18) with the appropriate $\hat{A}$ defines the DAMAS inverse problem. It is unique in that it or an equivalent equation must be the one utilized in order to disassociate the array itself from the sources being studied. Of course, the inverse problem must be solved in order to render $\hat{X}$.

DAMAS Inverse Problem Solution. Equation (18) is a system of linear equations. Matrix $\hat{A}$ is square (of size $N \times N$ ) and if it were nonsingular (wellconditioned), the solution would simply be $\hat{X}=\hat{A}^{-1} \hat{Y}$. However, it has been found for the present acoustic problems of interest that only for overly restricted resolution (distance between $n$ grid points) or noise region size (spatial expanse of the $N$ grid points) would $\hat{A}$ be nonsingular. Using Singular Value Decomposition (SVD) methodology ${ }^{20}$ for determining the condition of $\hat{A}$, it is found that for resolutions and region sizes of common interest in the noise source mapping problem in aeroacoustic testing that the rank of $\hat{A}$ can be quite low - often on the order of 0.25 and below. Rank here is defined as the number of linearly independent equations compared to the number of equations of Eq. (18), which is $N=$ number of grid points. This means there are generally very large numbers of "solutions". Equation (18) and the knowledge of the difficulty with equation rank were determined early in the present study. The SVD solution approach with and without regularization methodology ${ }^{21}$, special iterative solving methods such as Conjugate Gradient methods ${ }^{20}$, and others did not produce satisfactory results. Good results were ultimately obtained by a very simple tailored iterative method where a physically-necessary positivity constraint (making the problem deterministic) on the $X$ components could be applied smoothly in the iteration. This is described below.

A single linear equation component of Eq. (18) is

$A_{n 1} X_{1}+A_{n 2} X_{2}+\ldots+A_{n n} X_{n}+\ldots+A_{n N} X_{N}=Y_{n}$

With $A_{n n}=1$, this is rearranged to give 


$$
X_{n}=Y_{n}-\left[\sum_{n^{\prime}=1}^{n-1} A_{n n^{\prime}} X_{n^{\prime}}+\sum_{n^{\prime}=n+1}^{N} A_{n n^{\prime}} X_{n^{\prime}}\right]
$$

This equation is used in an iteration algorithm to obtain the source distribution $X_{n}$ for all $n$ between 1 and $N$ as per the following equation.

$$
\begin{aligned}
& X_{1}^{(i)}=Y_{1}-\left[0+\sum_{n^{\prime}=1+1}^{N} A_{1 n^{\prime}} X_{n^{\prime}}^{(i-1)}\right] \\
& X_{n}^{(i)}=Y_{n}-\left[\sum_{n^{\prime}=1}^{n-1} A_{n n^{\prime}} X_{n^{\prime}}^{(i)}+\sum_{n^{\prime}=n+1}^{N} A_{n n^{\prime}} X_{n^{\prime}}^{(i-1)}\right] \\
& X_{N}^{(i)}=Y_{N}-\left[\sum_{n^{\prime}=1}^{N-1} A_{N n^{\prime}} X_{n^{\prime}}^{(i)}+0\right]
\end{aligned}
$$

For the first iteration $(i=1)$, the initial values $X_{n}$ can be taken as zero or $Y_{n}$ (the choice appears to cause little difference in convergence rates). It is seen that in the successive determination of $X_{n}$, for increasing $n$, the values are continuously fed into the succeeding $X_{n}$ calculations. After each $X_{n}$ determination, if it is negative, its value is set to zero. Each iteration (i) is completed by like calculations, but reversed, moving from $n=N$ back to $n=1$. The next iteration $(i+1)$ starts again at $n=1$. Equation (24) is the DAMAS inverse problem iterative solution.

\section{APPLICATION SIMULATION AND CRITERIA}

Key Application Parameters. Figure 2 has identified important parameters in defining the solution requirements for DAMAS for a scanning plane. The array has a spatial extent defined by the "diameter" $D$. It is at a nominal distance $R$ from a scanning plane containing $N$ grid points, which represent beamforming focal points, as well as the $n$ locations of all the acoustic sources $X_{n}$ that influence the beamformed results $Y_{n}$. For a particular frequency, the array's beamformed output is shown projected on the plane as contour lines of constant output $Y$, in terms of $\mathrm{dB}$. The scanning plane has a height of $H$ and a width of $W$. The grid points are spaced $\Delta x$ and $\Delta y$ apart. Although not illustrated in Fig. 2, there are defined noise source sub-regions of size $\ell$ within the scanning plane (subsets of $X_{n}$ ), where details are desired. This relates to source resolution requirements and is considered below. For the scanning plane, the total number of grid points,

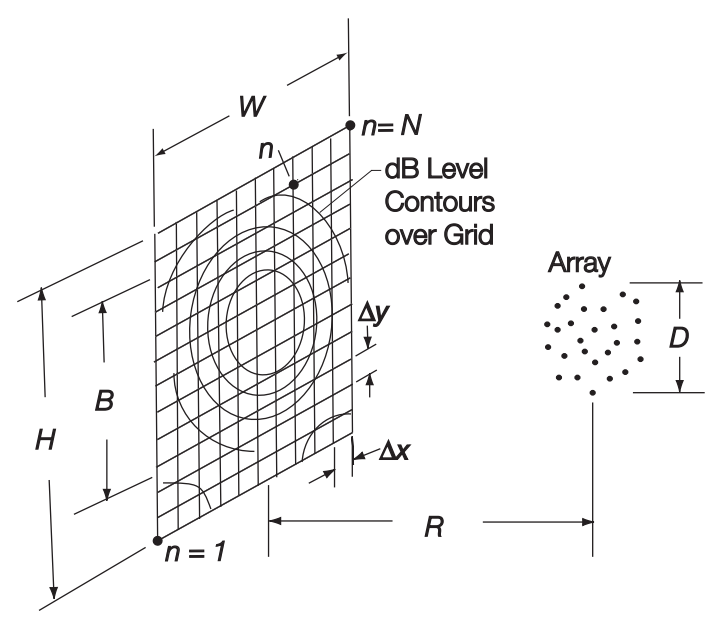

FIGURE 2 Key geometric parameters of the array and source region scanning plane.

$$
N=[(W / \Delta x)+1][(H / \Delta y)+1]
$$

The array beamwidth $B$ is defined as the "diameter" of the $3 \mathrm{~dB}$-down output of the array compared to that at the beamformed maximum response. For standard (STD) beamforming of Eq. (6),

$$
B \approx \text { const } \times(R / f D)
$$

For the SADA (Small Aperture Directional Array with a outer diameter of $D=.65$ feet) in a traditional QFF configuration $^{1}$ with $R=5$ feet, the beamwidth is $B \approx\left(10^{4} / f\right)$ in feet for frequency $f$ in Hertz. When using shading of Eq. (7), $B$ is kept at about $1 \mathrm{ft}$. for $10 \mathrm{kHz} \leq f \leq 40 \mathrm{kHz}$.

In the applications of this report, some engineering choices are made with regard to what should represent meaningful solution requirements for DAMAS source definition calculations. Because the rank of matrix $\hat{A}$ of Eq. (18) equals one when using the iterative solution Eq. (24), there is no definitive limitation on the spacing or number of grid points or iterations to be used. The parameter ratios $\Delta x / B$ (and $\Delta y / B$ ) and $W / B$ (and $H / B)$ appear to be most important for establishing resolution and spatial extent requirements of the scanning plane. The resolution $\Delta x / B$ must be small or fine enough such that individual grid points along with other grid points represent a reasonable physical distribution of sources. However, too fine of a distribution would require substantial solution iterative times and then only give more detail than is realistically feasible, or believable, from a beampattern 
which is too broad. On the other hand, too coarse of a distribution would render solutions of $\hat{X}$ which would reveal less detail than needed, and also which may be aliased (in analogy with FFT signal processing), with resulting false images. The spatial extent ratio $W / B$ (and $H / B)$ must be large enough to allow discrimination of mutual influence between the grid points. Because the total variation of level over the distance $B$ is only $3 \mathrm{~dB}$, it appears reasonable to require that $1<W / B$ (and $H / B$ ). One could extend $W / B$ (and $H / B$ ) substantially beyond one - such as to five or more. In the following simulations, resolution issues are examined for both a simple and a complicated noise source distribution. Two distributions types are considered because, as seen below with respect to $\ell / B$, source complexity affects source definition convergence. The simulations also serve as an introduction to the basic use of DAMAS.

Regarding execution efficiency of the DAMAS technique, it is noted that the per-iteration execution time of the algorithm depends solely on the total number of grid points employed in the analysis and not on frequency-dependent parameters. In general, the iteration time can be expressed by time $=C(2 N)^{2} i$, where $C$ is a hardware-dependent constant. A representative execution time is 0.38 seconds/iteration running a 2601-point grid on a $2.8-\mathrm{GHz}$, Linux-based Pentium 4 machine using Intel Fortran to compile the code. For this study, a Beowulf cluster consisting of nine $2.8 \mathrm{GHz}$ Pentium 4 machines was used to generate the figures shown subsequently.

Simple Source. In a traditional contour type presentation, the top left frame of Fig. 3(a) shows an array output based on standard (STD) processing methodology of Eq. (6), being plotted in terms of constant $\mathrm{dB}$ contours over a scanning plane. In this simulation, the SADA is placed 5 feet from the plane that is positioned through a typical model location. In terms of the aforementioned parameters, $H=W=50$ " and $\Delta x=\Delta y=1$ ". The resultant number of grid points is 2601 (underlying grid points are not shown in top left frame). With a chosen frequency of $10 \mathrm{kHz}$ and the beamforming of Eq. (6), $B \approx 12$ ", so $H / B=W / B=$ 4.17 and $\Delta x / B=0.083$. A single synthetic point source is placed at a grid point in the center of the plane, at $n=1301$. This is done by defining $X_{1301}$ to give $100 \mathrm{~dB}=10 \log X_{1301}$ and all other $\hat{X}$ values to zero in Eq. (18), and then solving for $\hat{Y}$. The values of $d B=10 \log Y_{n}$ are then contour plotted. This, as with real array test data, is the starting point for the use of DAMAS. Equation (18) is solved for $\hat{X}$ using Eq. (17) for $A_{n n^{\prime}}$, by way of Eq. (24), using $X_{n}=Y_{n}$ at the start

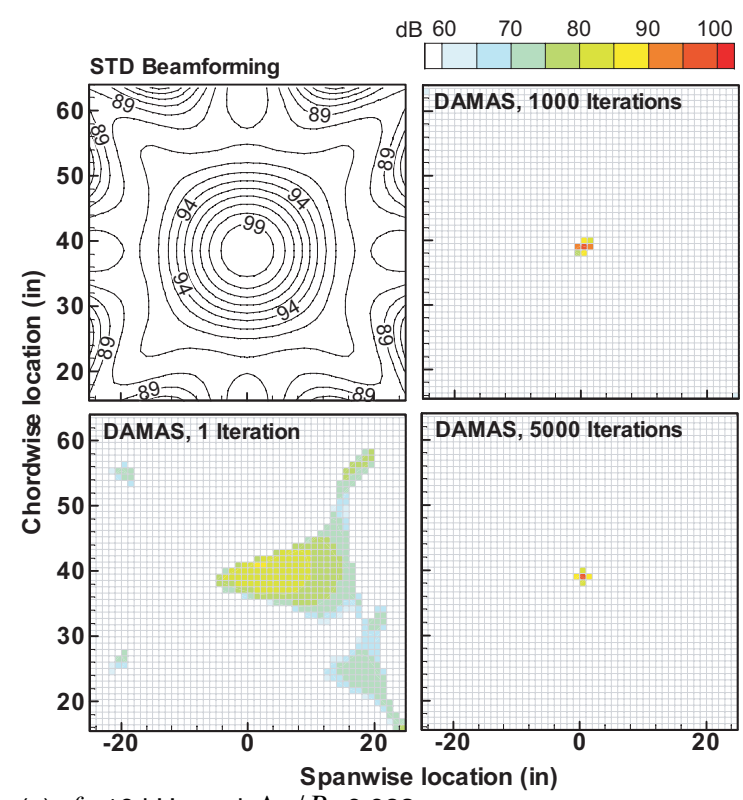

(a) $f=10 \mathrm{kHz}$ and $\Delta x / B=0.083$

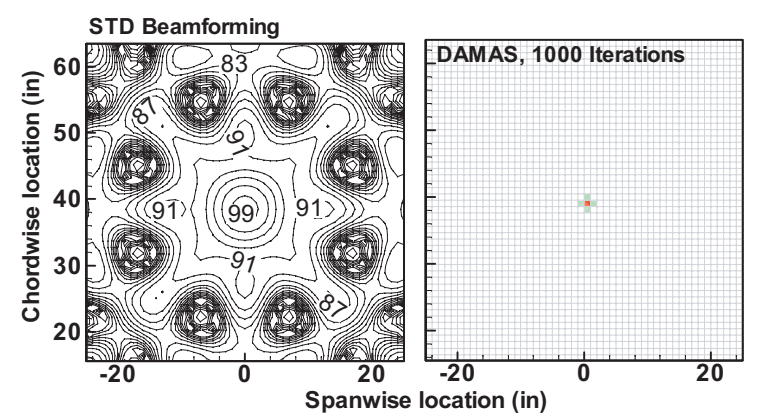

(b) $f=20 \mathrm{kHz}$ and $\Delta x / B=0.167$

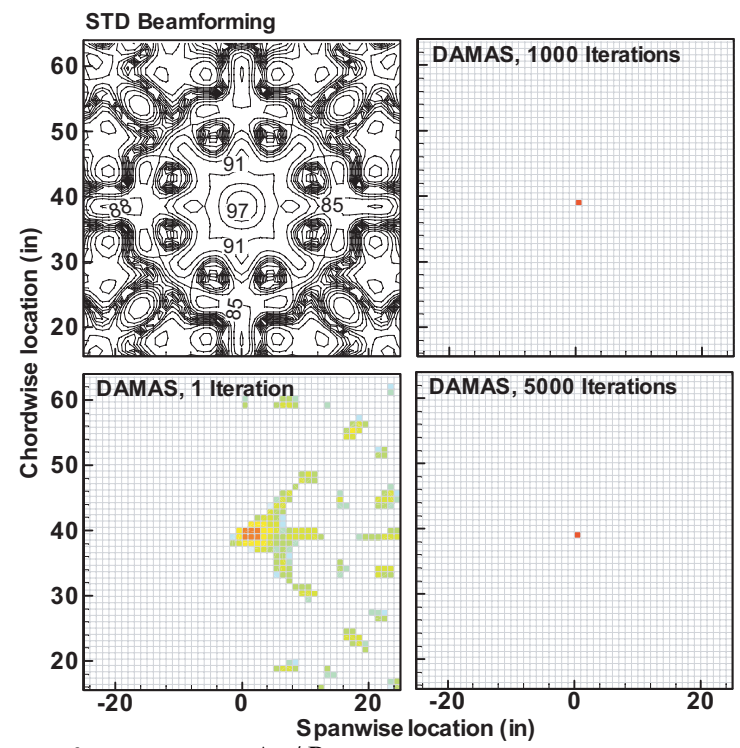

(c) $f=30 \mathrm{kHz}$ and $\Delta x / B=0.25$

FIGURE 3 Synthetic point source - SADA STD processing for 50 "x 50 " scanning plane that is 5' away. Resolution $\Delta x=1$ ". 


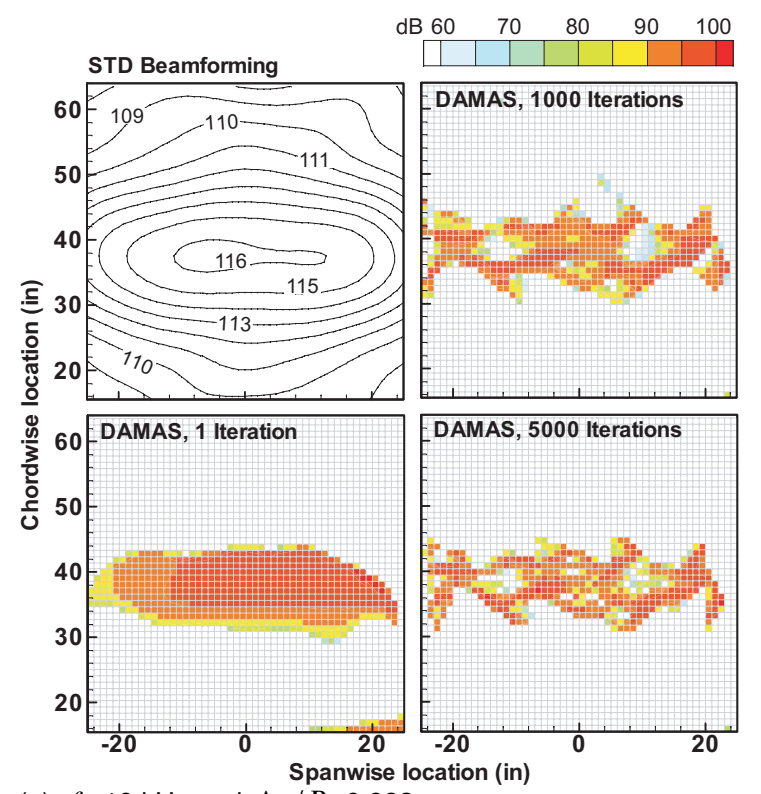

(a) $f=10 \mathrm{kHz}$ and $\Delta x / B=0.083$

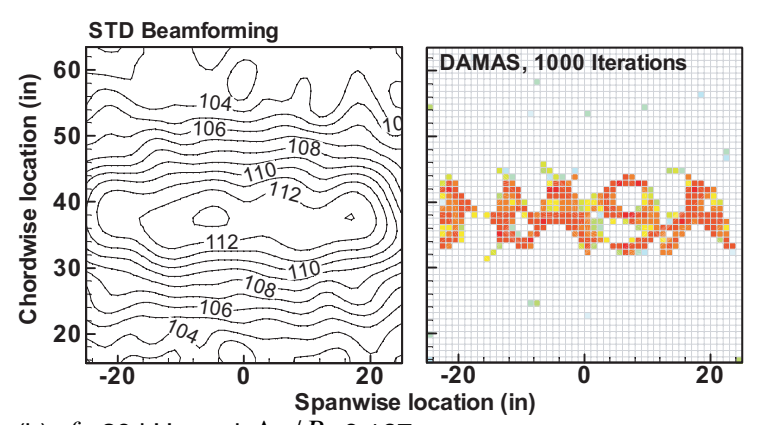

(b) $f=20 \mathrm{kHz}$ and $\Delta x / B=0.167$

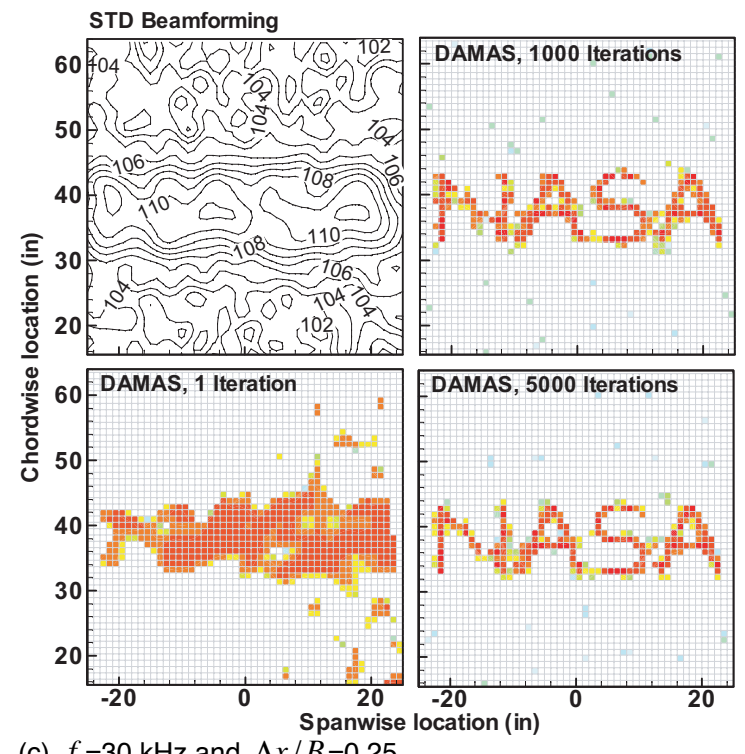

(c) $f=30 \mathrm{kHz}$ and $\Delta x / B=0.25$

FIGURE 4 NASA image source - same conditions as Fig. 3.

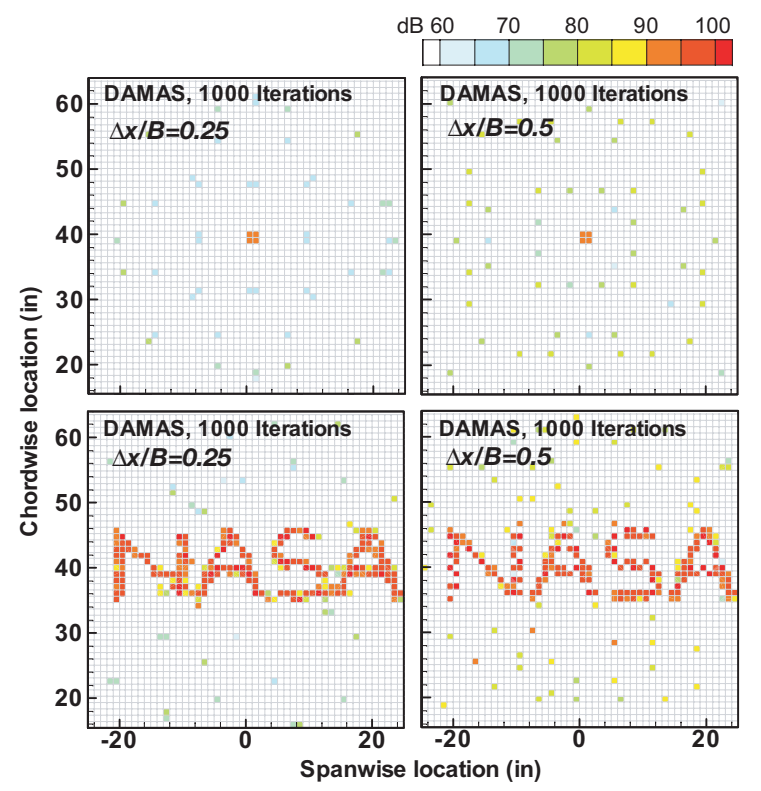

FIGURE 5 Spatial aliasing with point source and image shifted between grid points. DAMAS with 1000 iterations.

of the iteration. The bottom left frame of Fig. 3(a) shows the values of $X_{n}$ after one iteration $(i=1)$. Rather than showing contours, the presentation is one of $X_{n}$ values in terms of $\mathrm{dB}$ at the grid points. Each grid point is actually located at the bottom left corner of the "blocks", each of dimension $\Delta x=\Delta y=1$ ". In the top right and bottom right frames of Fig. 3 (a), the results after the one thousandth $(i=1000)$ and the five thousandth $(i=5000)$ iteration, respectively, are shown. At the highest iteration value, the original input value of $100 \mathrm{~dB}$ has been recovered within $0.1 \mathrm{~dB}$ and that the surrounding grid values over the plane are down in level by about $40 \mathrm{~dB}$, except for the adjoining grid points at about 15-20 dB down. At the lesser iteration numbers, although there is some spreading of the source region, the integrated (obtained by simple summing of values over the spread region) levels are very close to $100 \mathrm{~dB}$. One obtains $99.06 \mathrm{~dB}$ for 100 iteration (not shown in Fig. 3 (a)) and $100.03 \mathrm{~dB}$ for 1000 iterations.

The solution dependence on reducing the beamwidth $B$ by a factor of two $(\Delta x / B=0.167)$ is demonstrated in Fig. 3(b) where the frequency used is $20 \mathrm{kHz}$ using the same standard processing over the same grid. The contour pattern is similar, but contracted, as shown in the left frame. The DAMAS result for 1000 iterations is given in the right frame. Comparing this to the results of Fig. 3(a), it is seen that here a more exact solution is attained with substantially less iterations. One obtains at peak of $99.97 \mathrm{~dB}$, with all adjoining grid points lower by $27 \mathrm{~dB}$. 
The beamwidth $B$ is again reduced for Fig. 3(c), where the frequency used is $30 \mathrm{kHz}$, to obtain $\Delta x / B=0.25$. With the same result presentation as Fig. $3(a)$, it is seen that again much less iteration is needed to attain more exact results with larger $\Delta x / B$. At 1000 iterations, one obtains $100.00 \mathrm{~dB}$ at the peak, with adjoining grid points being lower by $61 \mathrm{~dB}$. Even at 100 iterations (not shown), the results compare well with 5000 iterations of Fig. 3(a).

Complicated Source. A more demanding simulation is shown in Fig. 4, where a distribution of $n$ locations was defined with the same $X_{n}$ values (each corresponding to $100 \mathrm{~dB}$ ) and others as zero. This gives a test of the solution procedure for a group of line source distributions. The scanning plane parameters, including the number of solution iterations, given for Fig. 4 are the same as those for Fig. 3. In Fig. 4(a) for $\Delta x / B=0.083$, the beamforming contour plot has an elongated appearance similar to one that would be expected for a single line source. But after using some iterations, one begins to see structure other than a single line source. Still, the image does not converge (it does not converge even after 30,000 iterations). In Fig. 4 (b), for $\Delta x / B=0.167$, a "very prominent" image emerges (the image is found to be recognizable even before 100 iterations). In Fig. 4(c), for $\Delta x / B=0.25$, all images are apparent. With regard to integrated power, it is found that when integrating (by summing grid point values), the total noise converges to the correct value rapidly with increasing iterations (within $1 \mathrm{~dB}$ after 16 iterations and within $.05 \mathrm{~dB}$ after 100 iterations) for all three $\Delta x / B$ values.

Comparing Figs. 3 and 4, it is seen that the degree of complexity of the source distributions does have an impact on the image convergence. It appears to be independent of the basic $\Delta x / B$ dependence demonstrated in Fig. 3. The source complexity resolution effect is quantified here by associating $\ell$, first, with a representative dimension of the closed portion of the letter "A" formed by the point sources $(\approx$ 6 ") and, second, with the overall letter dimensions $(\approx$ 11 "), and then relating these to beamwidth $B$. From this, and a subjective judgment of image quality, resolution of source region detail is only marginal for $\ell / B<1$, acceptable for $1 \leq \ell / B$, and good for $2<\ell / B$.

Spatial Aliasing from Source Distribution. It is seen above that coarser $\Delta x / B$ resolutions require less iterations to get the same "accuracy". And, this becomes even more true as the noise source becomes more complicated. A question is raised with regard to the potential error one may encounter if $\Delta x / B$ is made too large in real data cases where significant sources may be in-between chosen grid points (all line sources contain energy between grid points). For any such error, analogy can be made with the common data analysis subject of aliasing errors with respect to FFT sampling rates. No problems of this nature are possible in the previous simulations because all sources are collocated at the grid points. To evaluate the degree of the potential aliasing problem, the simulations of Figs. 3 and 4, are repeated with the noise sources being offset by one-half the distance between the grid points, thereby shifting the complete $Y$ contour map diagonally by that amount. Figure 5 shows the DAMAS results for $X_{n}$ with this offset for two of the $\Delta x / B$ cases, one with $\Delta x / B=0.25$, corresponding to the coarsest cases of Figs. 3 and 4, and one twice as large at $\Delta x / B=0.5$. If there were no aliasing problem, the position offset for the single source should cause the four adjoining points to be equal and sum to a total of $100 \mathrm{~dB}$, while the remaining scanning plane should have negligible summed levels. For $\Delta x / B=0.083$, $0.167,0.25,0.5,0.75$, and 1 , the summed four levels for 1000 iterations (and the summed levels in the remaining plane) are 100.0 (67.8), 100.1 (82.7), 100.2 (88.3), 100.5 (96.6), 99.8 (99.9), and $97.2(97.5) \mathrm{dB}$, respectively. For the NASA image, as well as for the single source, it is found that there is small observable aliasing effects for $\Delta x / B=0.083$ and 0.167 . However, it is seen in Fig. 5 that the NASA image brakes up to some degree for $\Delta x / B=0.25$, and to a larger extent for 0.5 . For $\Delta x / B=0.75$, the image is disrupted, and for $\Delta x / B=1$, it is destroyed. It should be kept in mind that these calculations represent limiting case tests for aliasing. Real data would be smoothed by the presence of other distributed sources. Still, it appears that to avoid aliasing problems, values of $\Delta x / B$ at or below 0.2 are recommended.

\section{EXPERIMENTAL APPLICATIONS}

In this section, experimental data from several airframe component noise studies conducted in Langley's QFF are re-examined with DAMAS. In these applications, DAMAS is not used with necessarily optimum resolution and scanning plane size. However, all cases fall at or near an acceptable range of $0.05 \leq \Delta x / B$ (and $\Delta y / B) \leq 0.2$. For consistency with the simulations, (except for the calibrator case) the same scanning plane and resolution sizes are used with the same resultant number of grid points. The number of iterations used for all is 1000 . In contrast with the simulations, the experimental results are presented in terms of one-third octave values, for the array using several different array beamforming methodologies, in order to compare to the results of the previous studies. 


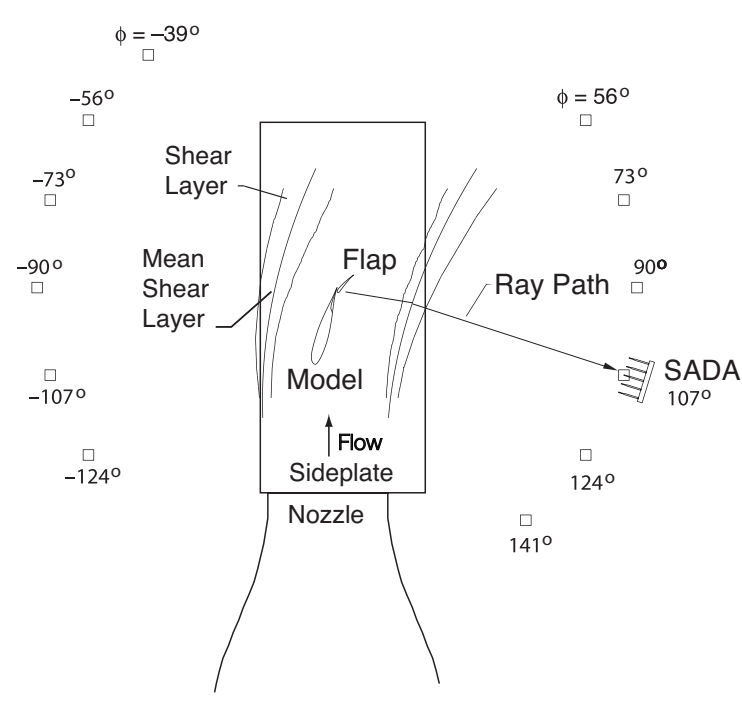

(a) Noise path from the flap edge to the SADA is illustrated.

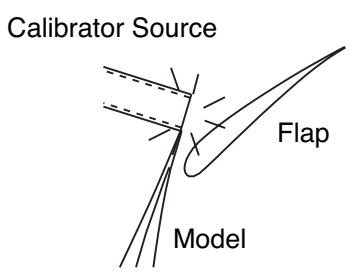

(b) Open end of calibrator source positioned next to flap edge.

FIGURE 6 Sketches of test set up for flap edge noise test and calibration.

Calibrator Source w/wo Flow. A sketch of the flap edge noise experimental setup is shown in Fig. 6(a). The airfoil main element is at $16^{\circ}$ angle-of-attack to the vertical plane. The SADA is shown positioned out of the flow. For this configuration ${ }^{1}$, the calibration test is performed using a noise source, comprised of an open end of a one-inch diameter tube, placed next to the flap edge, as shown in Fig. 6(b).

For the calibrator source operating with no tunnel flow $M=0$, Fig. 7(a) shows SADA response contours for standard (STD) processing with shading, Eq. (7), over a scanning plane positioned through the airfoil chordline. This corresponds to a frame of Fig. 7 (a) in Ref. 1. This is a one-third octave presentation for $10 \log Y$ for $f_{1 / 3}=40 \mathrm{kHz}$. The result was obtained by performing and summing 546 single-frequency beamforming maps (each with frequency resolution bandwidth of $\Delta f=17.44 \mathrm{~Hz}$ ). Note that with this array shading, only the inner SADA diameter of 1.95 inches is active. For this no-flow case, the convective and shear layer refraction terms are absent in the steering vector definition, Eqs. (4) and (5). The right frame of Fig. 7(a) shows the result for the rendered source $X$ distribution when DAMAS is applied, solving Eq. (18), using Eq. (19), by way of Eq. (24). The scanning plane used is $H=W=12$ ", in order to match that of Fig. 7(a) of Ref. 1. Consistent with the contour presentation, the DAMAS result is a one third octave presentation obtained by separately solving for the 546 separate bands and then summing. With $B=12$ " and a chosen $\Delta x=\Delta y=0.55$ ", one has a resolution of $\Delta x / B=0.046$ (which is close to the recommended lower limit of $0.05)$. The number of grid points is 441 and the number of iterations used is 1000 for each frequency.

Note that a characteristic of the DAMAS solution is the non-negligible amplitudes distributed at grid points around the border of the scanning planes in Fig. 7(b). This is a scanning plane "edge" effect that is found to occur only for experimental data, where noise in the scanning plane is influenced to some degree by sources outside (or extraneous to) the plane. DAMAS constructs noise distribution solutions on the scanning plane grid points totally based on whatever is measured by beamforming on those grid points. The edge effect was examined by expanding the scanning plane to eliminate any edge problem in the region of interest. A result almost identical to Fig. 7(a) was found over regions other than at the edge. Thus the edge effect has negligible impact on these results. This subject is dealt with subsequently for other applications.

In Ref. 1, a small rectangular integration region, shown by dashed lines in Fig. 7(a), was used to calculate an integrated value of $62.8 \mathrm{~dB}$. Correspondingly, for the present DAMAS result, one simply adds the pressure-squared values of the grid points within the source region. One obtains a value of 62.9 dB. Figure 7(b) shows the SADA response contour for the tunnel flow at $M=0.17$. Here, the convective and shear layer refraction terms are important in the steering vector definition. The integrated value from Ref. 1 is $58.1 \mathrm{~dB}$, whereas the DAMAS value is $57.3 \mathrm{~dB}$. It is seen by comparing the somewhat smeared image of Fig. 7(b) to 7(a) that the affect of the tunnel flow and the resultant turbulent shear layer is to spread the apparent noise region. The subject of turbulent shear layer noise scattering is analyzed in Refs. 1 and 22. The DAMAS result in Fig. 7(b) is of particular interest because, to the knowledge of the authors, it may be the first direct measure of spatial dispersion of noise due to turbulence scatter. 


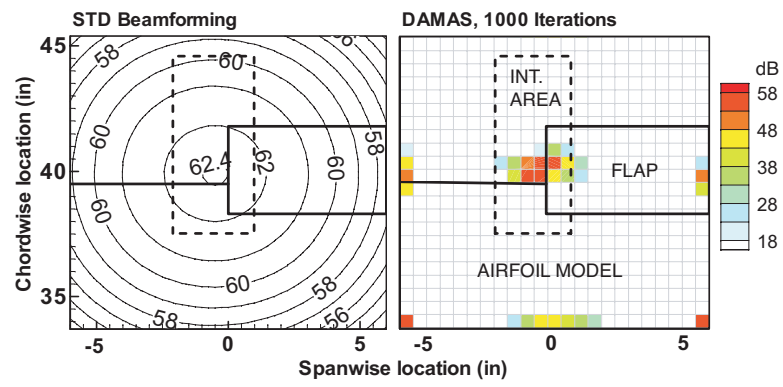

(a) $\mathrm{M}=0$, integrated level Ref.1 $62.8 \mathrm{~dB}$, DAMAS $62.9 \mathrm{~dB}$

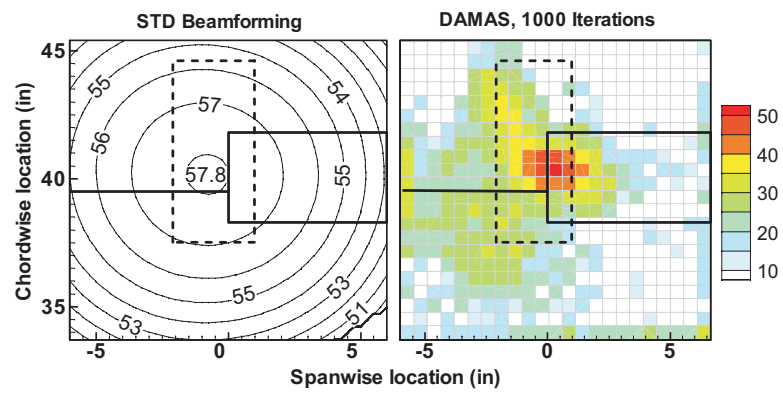

(b) $M=0.17$, integrated level Ref. $158.1 \mathrm{~dB}$, DAMAS $57.3 \mathrm{~dB}$

FIGURE 7 Calibrator source test, SADA shaded STD processing, $f_{1 / 3}=40 \mathrm{kHz}$ and $\Delta x / B=0.046$

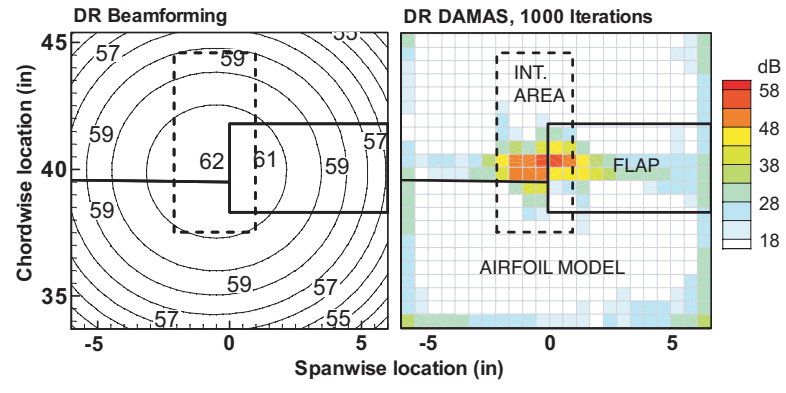

(a) $\mathrm{M}=0$, integrated level Ref.1 $62.2 \mathrm{~dB}$, DAMAS $61.6 \mathrm{~dB}$

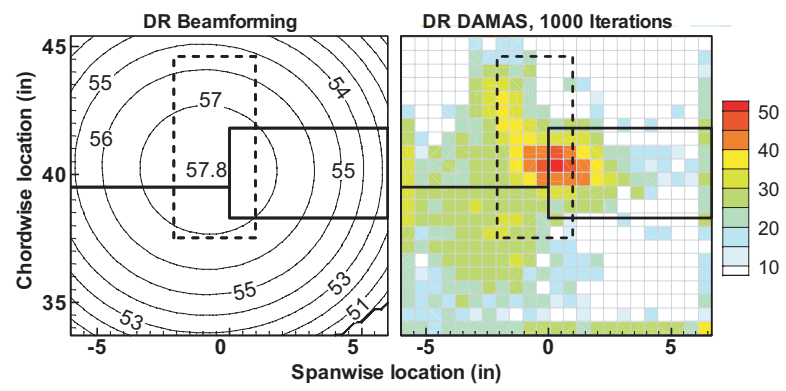

(b) $\mathrm{M}=0.17$, integrated level Ref. $57.9 \mathrm{~dB}$, DAMAS $56.9 \mathrm{~dB}$

FIGURE 8 Same conditions as Fig. 7, except shaded DR processing is used.
For the same test cases as Fig. 7, Fig. 8 shows the results when diagonal removal (DR), Eq. (9), is employed in the beamforming. Correspondingly, DAMAS is applied using Eq. (21) for $A_{n n^{\prime}}$. It is seen that although the DR processing modifies the $Y$ distributions, the $X$ source distributions and values are calculated to be almost identical to those of Fig. 7. As mentioned previously, DR processing has the advantage of removing the autospectra (and possible microphone noise contamination) from the processing, while still maintaining full rank for the solution equations.

Although it is beyond the scope of this paper to evaluate the use of DAMAS for different array designs than the SADA, a limited application using Large Aperture Directional Array (LADA) data produced good comparisons for a case corresponding to a frame of Fig. 7(a) in Ref. 1. The LADA has an outer diameter of $D=2.83$ feet, which is 4.35 times the size of SADA (17.4 times the active diameter of the SADA at $40 \mathrm{kHz}$ for shaded processing). For a similar calibration test to that of Fig. 7(a), but for array processing without shading, the integrated LADA value from Ref.1 is 60.3 $\mathrm{dB}$, and the corresponding DAMAS summed value is $61.4 \mathrm{~dB}$.

Trailing Edge and Leading Edge Noise Test. The test configuration ${ }^{4}$ where a NACA 63-216 airfoil, with a 16 " chord and $36^{\prime \prime}$ span, is positioned at $-1.2^{\circ}$ angle-of-attack to the vertical flow is shown in Fig. 9. The flap is removed and the cove is filled-in such as to produce a spanwise uniform sharp Trailing Edge (TE) of 0.005 ". Grit of size \#90 is distributed over the first $5 \%$ of the Leading Edge (LE) to ensure fully turbulent flow at the TE. The SADA position is at $\phi=90^{\circ}$. Figure 10 shows the array output over a scanning plane placed through the chordline. The scanning plane of size $H=W=50$ " extends "beyond" the sideplates that hold the 36" span airfoil. The sideplate regions as seen from the viewpoint of the array, to the left of the -18 " spanwise location and to the right of the 18 " location in Fig. 10, represent reflected source regions.

The array output in Fig. 10 is presented for four one-third octave frequencies for STD processing with shading, Eq. (7). As before, individual frequency results are processed and are then summed to obtain the results shown. It is seen that for the $f_{1 / 3}=3.15 \mathrm{kHz}$ case, that the most intense region is just aft of the airfoil TE. As frequency is increased, the intense regions appear to first concentrate near the TE, and then to shift towards the LE. 


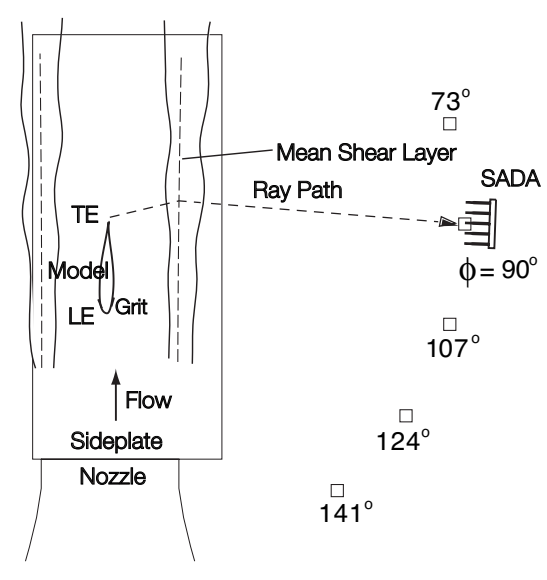

FIGURE 9 Sketch of test set up for TE and LE noise test.

Figure 11 presents DAMAS results corresponding to Fig. 10. For $f_{1 / 3}=3.15 \mathrm{kHz}, \Delta x=\Delta y=1.8$ " is used to obtain the chosen lower-limit resolution of $\Delta x / B=0.047$. For $8,12.5$, and $20 \mathrm{kHz}$, the chosen $\Delta x=\Delta y=1 "$ give $\Delta x / B=0.066,0.083$, and 0.083 , respectively, for shaded beamforming. The results shown appear to very successfully reveal noise source distributions, even those not apparent from Fig. 10. The TE and LE line sources are particularly well defined. The images at and beyond \pm 18 in. are model-sideplate noises and/or sideplate reflections. There are apparent phantom images, particularly aft of the TE and around the edges of the scanning plane. These are addressed below.

Figures 12 and 13 correspond to Figs. 10 and 11 , except that DR processing is used for beamforming, Eq. (9), and for DAMAS, Eq. (21). It is seen that although the beamforming contours differ significantly, the source distributions essentially match. The exception is that the DR results appear to produce cleaner DAMAS results, with much of the phantom images removed. That is, the apparent source distributions over regions away from surfaces where no "real" sources are likely to exist are significantly diminished. Also removed with DR is an "apparent" LE noise source distribution from the result of 3.15 $\mathrm{kHz}$. Considering that the present STD method results are to some degree contaminated with turbulence buffeting microphone self noise, the DR results are considered more correct. (This conclusion is supported by evidence evaluated in Ref. 5.)

Also present in Figs. 11 and 13 are edge effects as are found in and discussed for Figs. 7 and 8. The edge effects can be readily eliminated by expanding the scanning frame beyond the regions of strong sources, thereby reducing the edge amplitudes and thus any potential influence on the regions of interest. This has been verified but this is not shown here, as the edge effect's presence in Figs. 11 and 13 is instructive. For example, an area where the edge effect appears to negatively affect DAMAS results is the sideplate region on the left side near the LE (chordwise location 26 in. and spanwise location -21 in.). The strong array responses (Figs. 10 and 12) at that location are not correspondingly represented by the DAMAS source distributions in that region. Instead, DAMAS puts strong sources along the scanning plane edge and the LE corner to explain the array response. (Note that it is well recognized that the array response over such a corner location may well be influenced by reflected (and thus correlated) noise sources, whereas the DAMAS modeling is based on an equivalent statistically independent source distribution. The edge effect is unrelated to this modeling/reality physical difference. Such reflections undoubtedly cause strengthening or weakening and/or shifting of apparent sources, but it would not cause source concentration along the edges.) Still, even with the scanning plane edge effect, away from the edges the TE and LE noise source regions are unaffected and the following noise spectra serve to verify this.

Previously ${ }^{4}$, TE noise spectra were determined from amplitudes of the array response at the center of the TE, along with a transfer function based on an assumed line source distribution. Also, corresponding spectra from the LE noise region were determined to show grit-related LE noise, which due to beamwidth characteristics were contaminated by TE noise at low frequencies. Figure 14 shows one-third octave spectra (per foot) curves from Fig. 13 of Ref. 4 for the test conditions corresponding to Figs. 10-13 above. These spectra are compared to spectra of TE noise and LE noise determined from DAMAS using both STD and DR methods. These results are determined by simply summing the pressure-squared values of each grid point within the rectangular box region surrounding the TE and LE regions shown superimposed in Figs. 11 and 13. The region's spanwise length is 2.5 feet. The sums are divided by 2.5 to put the spectral results on a per-foot basis. The spectral comparisons are quite good and serve as a strong validation for the different analyses. Where low-frequency results of DAMAS are not plotted, the integration regions lacked contributions (not surprising with the very large beamwidths $B$ ). The spectra are seen to agree well with the results of Ref. 4 over parts of the spectra where each source is dominant. Of course in the spectra shown from Ref. 4, as in the beamformed solutions of Figs. 10 and 12, the 

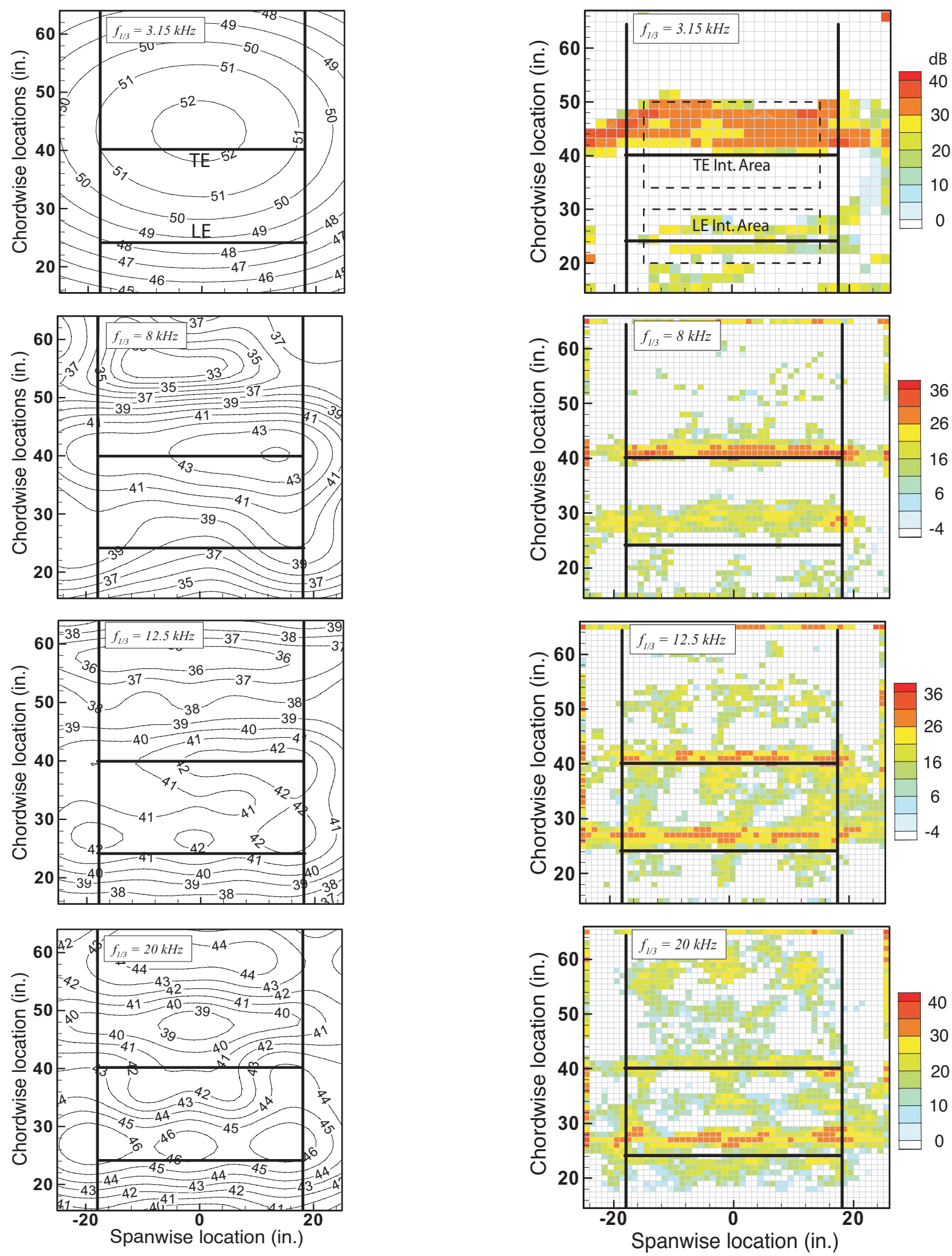

FIGURE 10 SADA response contours for Shaded STD processing - TE and LE noise test.

FIGURE 11 DAMAS results for Shaded STD processing - TE and LE noise test. Corresponding to Fig. 10. 

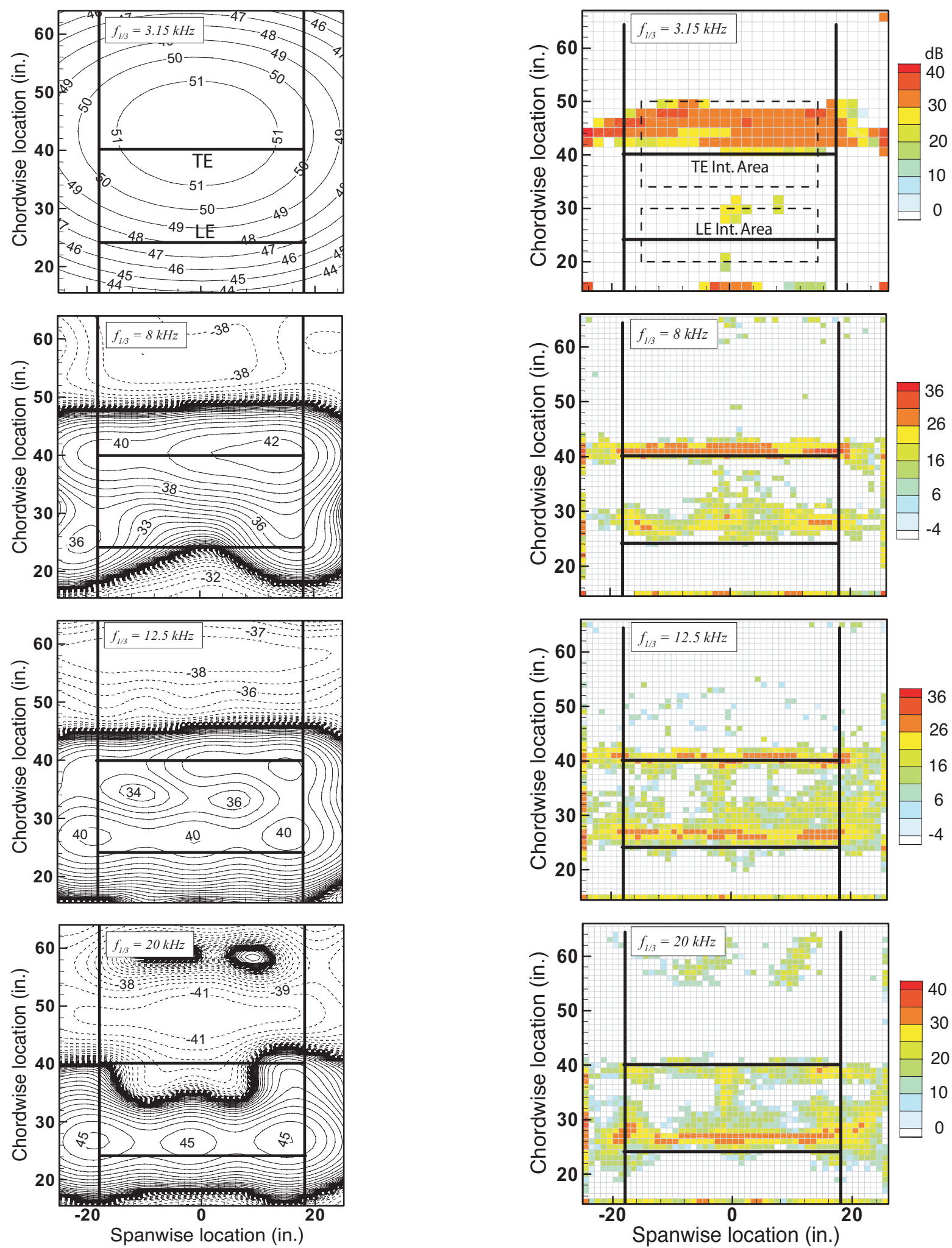

FIGURE 12 SADA response contours for Shaded DR processing - TE and LE noise test.

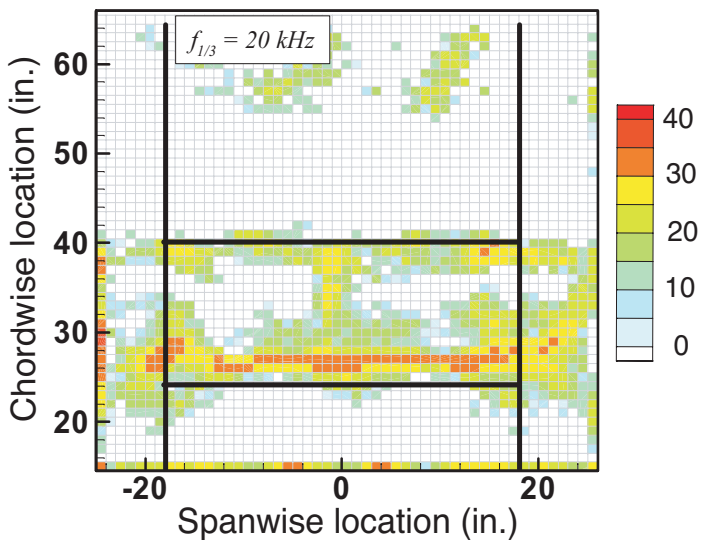

FIGURE 13 DAMAS results for Shaded DR processing - TE and LE noise test. Corresponding to Fig. 12. 


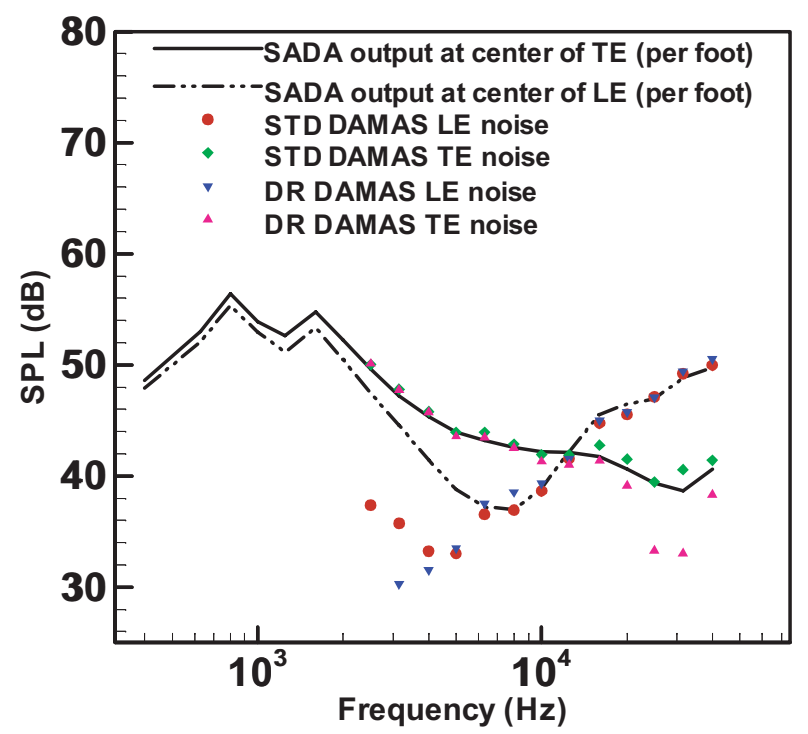

FIGURE 14 Comparison of one-third octave spectra from TE and LE noise measurements from Ref. 4 and reprocessing by DAMAS.

TE and LE noise region amplitudes are contaminated by the mutual influence of the other source over different parts of the spectra. The present DAMAS results exclude such interference.

Slat Noise Test. The slat configuration ${ }^{3}$ tested in the QFF is achieved by removing the flap, filling the flap cove (as for the TE noise test above), removing the grit boundary layer trip at the LE, tilting the airfoil main element to $26^{\circ}$ from vertical, mounting the slat, and setting the slat angle and gap. The large $26^{\circ}$ angle is required to obtain proper aerodynamics about the slat and LE region ${ }^{3}$. Figure 15 shows the airfoil / slat in the deflected open jet, with the SADA positioned at $\phi=107^{\circ}$.

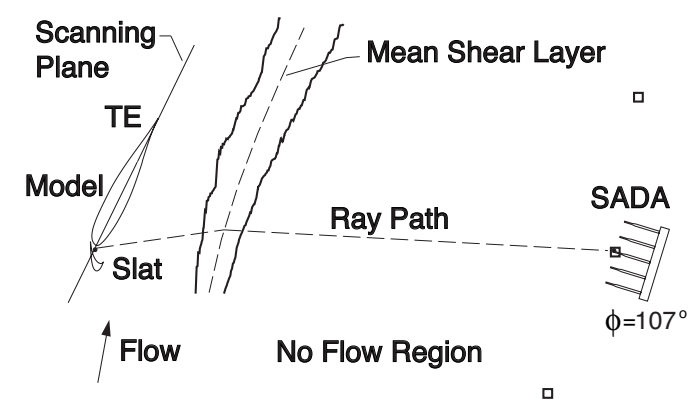

FIGURE 15 Sketch of test set up for slat noise test

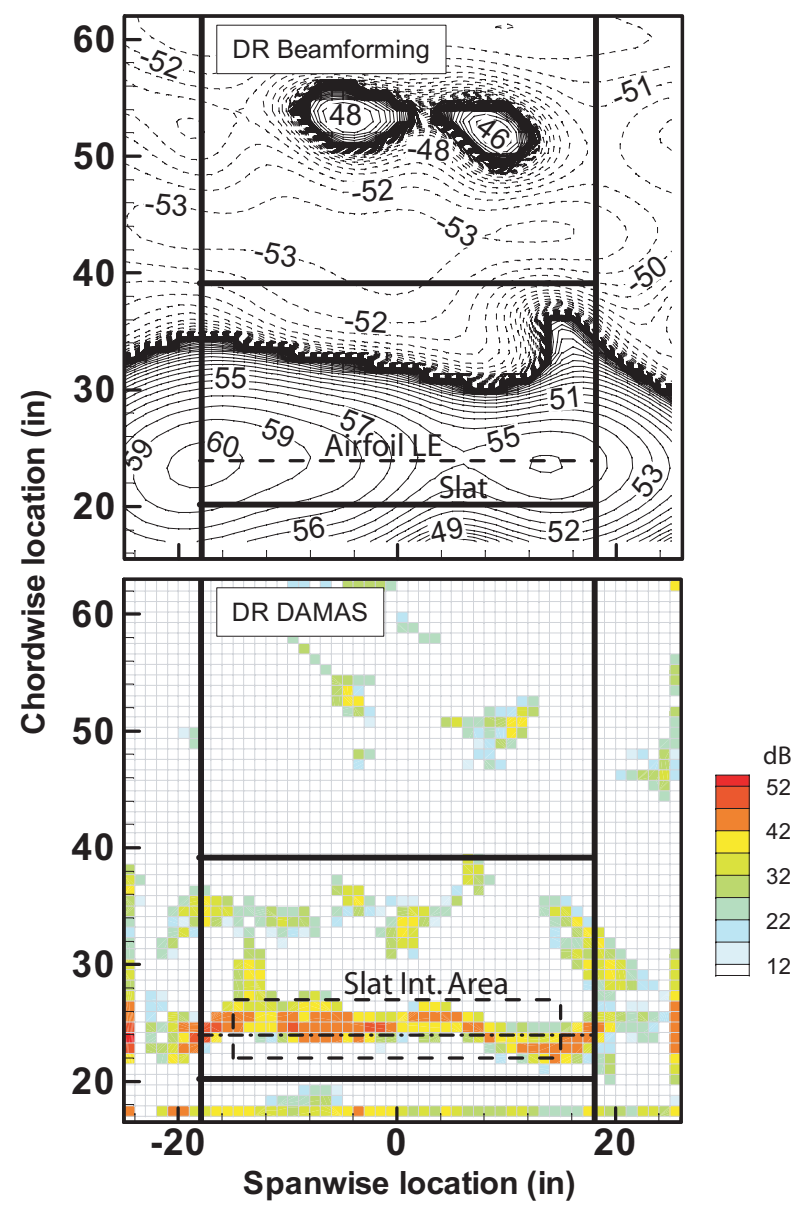

FIGURE 16 Slat noise test, SADA Shaded DR processing, $f_{1 / 3}=20 \mathrm{kHz}$ and $\Delta x / B=0.083$.

Figure 16 shows the array output and corresponding DAMAS result for $f_{1 / 3}=20 \mathrm{kHz}$ using shaded DR processing over the scanning plane through the airfoil. The distributed slat noise is seen to be well identified. There are higher levels toward the left side of the slat, likely due to a model /model mount irregularity. The aforementioned scanning plane edge effect is seen around the edge of the DAMAS presentation, and it likely has a mild impact on the source definition details at this left side. Away from the edge, the slat noise is generally uniform. The amplitude of the slat noise is determined by summing across the span within the integration box shown. For this one-third octave band, the DAMAS level of 57.9 $\mathrm{dB}$ (per foot) compares with $59.1 \mathrm{~dB}$ found by Ref. 3, which used an approximate procedure involving the array output at the slat center and a derived transfer function (similar to the procedure of Ref. 4 for TE noise). 


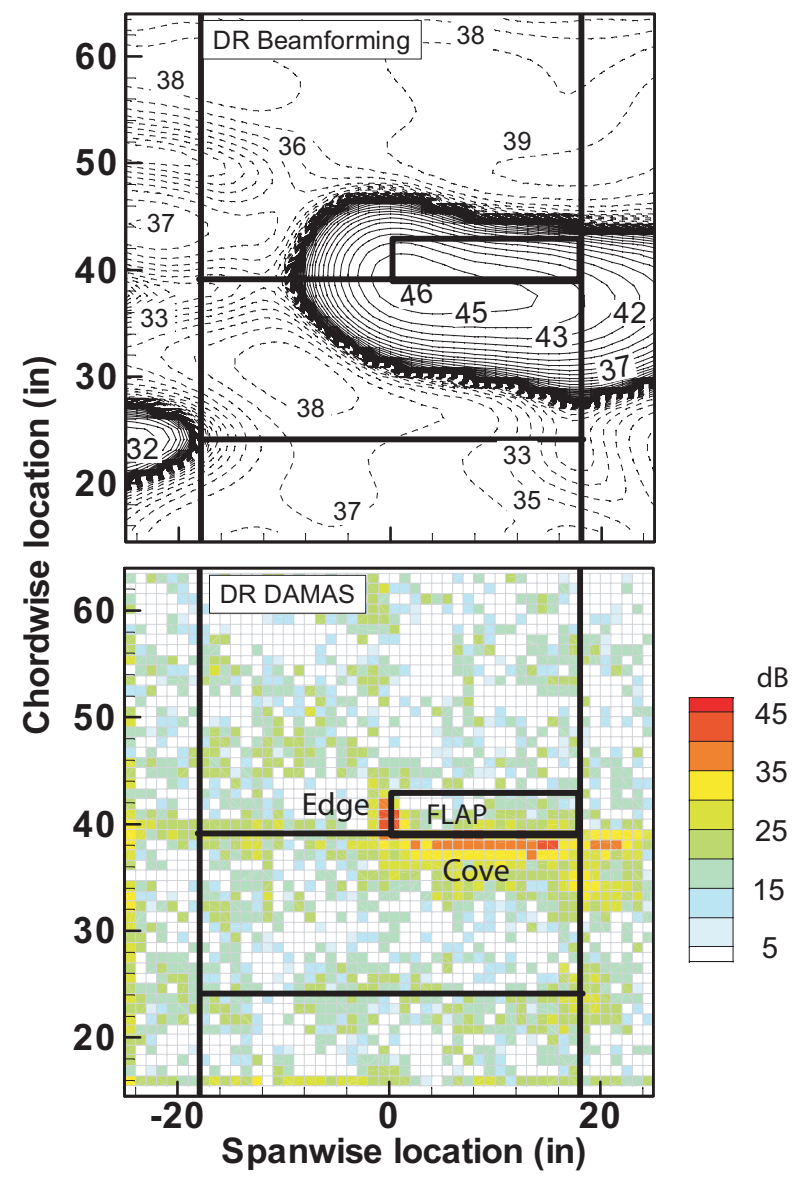

FIGURE 17 Flap noise test, SADA Shaded DR processing, $f_{1 / 3}=20 \mathrm{kHz}$ and $\Delta x / B=0.083$.

Flap Edge and Flap Cove Noise Test. The flap edge noise test configuration is shown in Fig. 6(a) for the SADA. The flat edge flap test condition of $29^{\circ}$ flap angle and $M=0.11$ is reexamined for a one-third octave frequency band of $f_{1 / 3}=20 \mathrm{kHz}$. Figure 17 shows beamforming contours and DAMAS results for shaded DR processing over the scanning plane placed through the airfoil chordline. The DAMAS results appear to successfully isolate the flap edge noise from substantial flap cove noise. By using a similar rectangular integration region, shown in Figs. 7 and 8, one finds a level of $44.6 \mathrm{~dB}$ for the flap edge noise. This compares to $47.5 \mathrm{~dB}$ for a spectrum level determined for this flap edge noise case in Ref. 2. In that spectrum, this frequency corresponds to a localized spectral hump. These present results show that the cove noise contributed to the higher level measured. DAMAS is seen to allow one to readily separate and quantify these cove and flap edge contributions, where previously this was not possible.

\section{CONCLUSION}

DAMAS represents a radical step in array processing capabilities. It can replace traditional presentations of array results and make the array a much more powerful measurement tool than is presently the case. The DAMAS equation $\hat{A} \hat{X}=\hat{Y}$ is a unique equation that relates a classical beamformed array result $\hat{Y}$ with the source distribution $\hat{X}$. The sources are taken as distributions of statistically independent noise radiators, as does traditional array processing/integration analysis. DAMAS does not add any additional assumption to the analysis. It merely extracts the array characteristics from the source definition presentation. The iterative solution for $\hat{X}$ is found to be robust and accurate. Numerical application examples show that the actual rate and accuracy at which solutions converge depend on chosen spatial resolution and evaluation region sizes compared to the array beamwidth. Experimental archival data from a variety of prior studies are used to validate DAMAS quantitatively. The same algorithm is found to be equally adept with flap edge/cove, trailing edge, leading edge, slat, and calibration noise sources.

\section{REFERENCES}

1. Brooks, T.F. and Humphreys, W.M., Jr., "Effect of Directional Array Size on the Measurement of Airframe Noise Components", AIAA Paper 99-1958, 1999.

2. Brooks, T.F. and Humphreys, W.M., Jr., "Flap Edge Aeroacoustic Measurements and Predictions", Journal of Sound and Vibration, Vol. 261, pp. 31-74, 2003.

3. Mendoza, J. M., Brooks, T. F. and Humphreys, W. M., "Aeroacoustic Measurements of a Leading Edge Slat Configuration", International Journal of Aeroacoustics, pp. 241-274, Vol. 1 (3), 2002.

4. Hutcheson, F.V. and Brooks, T.F., "Measurement of Trailing Edge Noise Using Directional Array and Coherent Output Power Methods", International Journal of Aeroacoustics, pp. 329-354. Vol. 1 (4), 2002.

5. Hutcheson, F.V. and Brooks, T.F., "Effects of Angle of Attack and Velocity on Trailing Edge Noise", AIAA Paper 2004-1031.

6. Oerlemans, S. and Sijtsma, P., "Determination of Absolute Levels from Phased Array Measurements Using Spatial Source Coherence", AIAA 2002-2464.

7. Sijtsma, P. and Holthusen, H., "Corrections for Mirror Sources in Phased Array Processing Techniques", AIAA Paper 2003-3196. 
8. Dougherty, R.P. and Stoker, R.W., "Sidelobe Suppression for Phased Array Aeroacoustic Measurements," AIAA Paper 98-2242, $4^{\text {th }}$ AIAA/CEAS Aeroacoustics Conference, Toulouse, France, June 2-4, 1998.

9. Hogbom, J.A., "Aperture Synthesis with a non-regular distribution of Interferometer Baselines", Astronomy and Astrophysics Supplements, Vol. 15, pp. 417-426, 1974.

10. Wang, Y., Li, J., Stoica, P., Sheplak, M., and Nishida, T., "Wideband RELAX and Wideband CLEAN for Aeroacoustic Imaging”, AIAA Paper 2003-3197, 2003.

11. Li, J. and Stoica, P., "Efficient Mixed-Spectrum Estimation with Applications to Target Feature Extraction", IEEE Transactions on Signal Processing, Vol. 44, pp. 281-295, 1996.

12. Wang, Z., Li, J., Nishida, T., and Sheplak, M., "Robust Capon Beamformers for Wideband Acoustic Imaging", AIAA Paper 2003-3198, 2003.

13. Dougherty, R.P., " Beamforming in Acoustic Testing", Aeroacoustic Measurements, T.J. Mueller (Ed.), Springer-Verlag, New York, 2002.

14. Blacodon,D. and Elias, G., "Level Estimation of Extended Acoustic Sources Using an Array of Microphones", AIAA Paper 2003-3199, 2003.

15. Brooks, T.F. and Humphreys, W.M., Jr., "Deconvolution Approach for the Mapping of Acoustic Sources (DAMAS), NASA Invention Disclosure", LAR-16907-1, NASA Langley Research Center, Hampton, VA, April 2004.

16. Humphreys, W. M., Brooks, T. F., Hunter, W. W. and Meadows, K. R., "Design and Use of Microphone Directional Arrays for Aeroacoustics Measurements", AIAA Paper 98-0471, 1998.

17. Amiet, R. K.," Refraction of Sound by a Shear layer", Journal of Sound and Vibration, Vol. 58 (3), pp. 467482, Sept. 1978.

18. Brooks, T.F., Marcolini, M.A., and Pope, D.S., "A Directional Array Approach for the Measurement of Rotor Noise Source Distributions with Controlled Spatial Resolution," Journal of Sound and Vibration, Vol. 112, No. 1, pp. 192-197, 1987.

19. Marcolini, M.A., and Brooks, T.F., "Rotor Noise Measurement Using a Directional Microphone Array," Journal of the American Helicopter Society, pp. 11-22, 1992.

20. Press, W.H., Teukolsky, S.A., Vetterling, W.T., and Flannery, B.P., Numerical Recipes in FORTRAN, The Art of Scientific Computing, $2^{\text {nd }}$ Ed., Press Syndicate of the University of Cambridge, Cambridge, UK, 1992.
21. Vogel, C.R., Computational Methods for Inverse Problems, Society for Industrial and Applied Mathematics, Philadelphia, PA, 2002.

22. Dougherty, R.P., "Turbulent Decorrelation of Aeroacoustic Phased Arrays: Lessons from Atmospheric Science and Astronomy," AIAA-2003-3200, 9 $9^{\text {th }}$ AIAA/CEAS Aeroacoustics Conference \& Exhibit, Hilton Head, S.C., May 12-14, 2003. 